\title{
Transforming growth factor- $\beta 1$ induces bronchial epithelial cells to mesenchymal transition by activating the Snail pathway and promotes airway remodeling in asthma
}

\author{
ZHAO-CHUAN YANG ${ }^{1}$, MING-JI YI ${ }^{1}$, NI RAN ${ }^{1}, \mathrm{CHONG}_{\mathrm{WANG}}{ }^{2}, \mathrm{PENG} \mathrm{FU}^{1}$, \\ XUE-YING FENG ${ }^{1}$, LEI XU ${ }^{2}$ and ZHENG-HAI QU ${ }^{2}$ \\ Departments of ${ }^{1}$ Child Health Care and ${ }^{2}$ Pediatrics, The Affiliated Hospital of Qingdao University Medical College, \\ Qingdao, Shandong 266003, P.R. China
}

Received May 18,2013; Accepted September 4, 2013

DOI: $10.3892 / \mathrm{mmr} .2013 .1728$

\begin{abstract}
Airway remodeling is characterized by airway wall thickening, subepithelial fibrosis, increased smooth muscle mass, angiogenesis and an increase in mucous glands, which may lead to a chronic and obstinate asthma with pulmonary function depression. In the present study, we observed substantially thickened lung tissue with extensive fibrosis in ovalbumin-sensitized mice, which was interrelated with transforming growth factor- $\beta 1$ (TGF- $\beta 1$ ) expression in bronchoalveolar lavage fluid. In vitro experiments further demonstrated that TGF- $\beta 1$ resulted in epithelial-mesenchymal transition (EMT) in bronchial epithelial cells, which was characterized by the expected decrease in E-cadherin expression and the increase in vimentin and $\alpha$-smooth muscle actin expression, as well as the associated increase in Snail expression at mRNA and protein levels. Furthermore, the downregulation of Snail by small interfering RNA (siRNA) attenuated the TGF- $\beta 1$-induced EMT-like phenotype. Of note, a significantly increased synthesis of fibronectin was observed following TGF- $\beta 1$ treatment, which further supported the hypothesis that EMT is a pivotal factor in peribronchial fibrosis. In combination, the results indicated that myofibroblasts deriving from bronchial epithelial cells via EMT may contribute to peribronchial fibrosis and that Snail may be an important factor in this phenomenon.
\end{abstract}

\section{Introduction}

Asthma is a common chronic inflammatory disease with an incidence that has markedly increased over the past two

Correspondence to: Dr Zheng-Hai Qu, Department of Pediatrics, The Affiliated Hospital of Qingdao University Medical College, 16 Jiangsu Road, Qingdao, Shandong 266003, P.R. China

E-mail: houjibofa20080808@163.com

Key words: airway remodeling, asthma, bronchial epithelial cells, Snail, transforming growth factor- $\beta 1$, epithelial-mesenchymal transition decades. In addition, the chronic inflammation apparent in a given patient is often associated with the remodeling of the airway structure, which may impair lung function $(1,2)$. These changes include peribronchial fibrosis, fibroblast proliferation and conversion to myofibroblasts, and smooth muscle hypertrophy (3). Myofibroblasts have been suggested to be pivotal factors in the pathogenesis of peribronchial fibrosis. However, the origin of the myofibroblast, the primary effector cell of peribronchial fibrosis, has not yet been elucidated. Three hypotheses have been proposed regarding the cellular origin of the myofibroblast. The first, and historically most prevalent, hypothesis postulates that resident peribronchial fibroblasts respond to a variety of stimuli during fibrogenic responses and differentiate into myofibroblasts. The second hypothesis postulates that myofibroblasts are derived from bone marrow progenitor cells (4), while the third possible source of fibroblasts and/or myofibroblasts to be proposed involves epithelial cells, through the process of epithelial-mesenchymal transition (EMT) (5).

EMT is an orchestrated series of events, in which differentiated epithelial cells undergo a phenotypic transition to mesenchymal cells, often fibroblasts and myofibroblasts $(5,6)$. During EMT, the epithelial cells lose intracellular junctions, leading to dissociation from the surrounding cells, acquire mesenchymal-like characteristics and become able to migrate away from the original location (7). This important process was initially recognized during embryonic development and has more recently been identified in tumor progression and organ fibrosis (8). To date, studies have suggested that kidney proximal tubule epithelial cells undergo EMT to induce interstitial fibrosis in progressive renal disease (9). In the fibrotic kidney, $\sim 36 \%$ of new fibroblasts arise from tubular epithelial cells (10). EMT may be triggered by different signalling molecules, such as transforming growth factor- $\beta 1$ (TGF- $\beta 1$ ), epidermal growth factor, fibroblast growth factor, hepatocyte growth factor and bone morphogenetic proteins (11).

TGF- $\beta 1$ is a profibrotic cytokine that has been indicated to be an important factor promoting the structural changes of airway remodeling in asthma (12). Snail, a zinc-finger transcription factor, has been characterized as a key EMT regulator (13). It has been shown that Snail binds to specific DNA sequences, known as E-boxes, in the promoter of the E-cadherin gene and 
represses the transcription of E-cadherin (14). Therefore, the downregulation of the cell-cell adhesion protein E-cadherin has been considered to be characteristic of EMT. Knockout mice deficient in Snail die at gastrulation due to a failure to undergo a complete EMT process, which leads to the formation of an abnormal mesodermal layer that maintains E-cadherin expression. In certain epithelial tumor cell lines, Snail-regulated EMT promotes cell motility and invasion $(15,16)$. An inverse correlation between E-cadherin and Snail expression has been observed in cultured epithelial lines established from breast cancer, pancreatic carcinoma and colon cancer. The silencing of Snail by stable RNA interference in epithelial cells was shown to attenuate the complete EMT, which was associated with the upregulation of E-cadherin, the downregulation of mesenchymal markers and the inhibition of invasion (17).

In the present study, we showed that bronchial epithelial cell transformation into myofibroblasts was associated with airway remodeling in asthma and that Snail transcription factor may be essential in TGF- $\beta 1$-mediated EMT in bronchial epithelial cells. These data suggested that bronchial epithelial cells may be the source of myofibroblasts in peribronchial fibrosis and may promote airway remodeling in asthma.

\section{Materials and methods}

Reagents. The chicken egg ovalbumin (OVA) used in the study was purchased from Sigma-Aldrich (St. Louis, MO, USA). E-cadherin, $\alpha$-smooth muscle actin ( $\alpha$-SMA), vimentin, fibronectin, Snail, glyceraldehyde 3-phosphate dehydrogenase (GAPDH) and secondary antibodies were purchased from Santa Cruz Biotechnology, Inc. (Santa Cruz, CA, USA). TGF- $\beta 1$ and a TGF- $\beta 1$ enzyme-linked immunosorbent assay (ELISA) kit were obtained from R\&D Systems (Minneapolis, MN, USA), while any other laboratory reagents were purchased from Sigma.

Cell line and culture. The 16HBE human bronchial epithelial cells used in this study were obtained from the Cancer Research Institute of Beijing, China. The cells were cultivated in T75 tissue culture flasks in RPMI-1640 supplemented with $10 \%$ fetal bovine serum (FBS), $100 \mathrm{IU} / \mathrm{ml}$ penicillin, $100 \mu \mathrm{g} / \mathrm{ml}$ streptomycin, $2 \mathrm{mM}$ L-glutamine and $20 \mathrm{mM}$ hydroxyethyl piperazine ethanesulfonic acid, and incubated in a humidified incubator containing $5 \% \mathrm{CO}_{2}$ at $37^{\circ} \mathrm{C}$.

Sensitization and antigen challenge. Twenty-four healthy female BABL/c mice, weighing 18-24 g, were randomly divided into the normal control and asthma groups. The mouse model of asthma was established using OVA. The mice were sensitized on days 0,7 and 14 by intraperitoneal injection of $20 \mu \mathrm{g}$ OVA emulsified in $1 \mathrm{mg}$ aluminum hydroxide, in a total volume of $0.2 \mathrm{ml}$. Seven days subsequent to the final sensitization, the mice were exposed to $1 \%$ OVA aerosol for $\leq 30 \mathrm{~min}$ every other day for eight weeks. The 1\% OVA aerosol was generated using a compressed air atomizer driven by filling a Perspex cylinder chamber (diameter $50 \mathrm{~cm}$, height $50 \mathrm{~cm}$ ) with a nebulized solution. Saline was used in the control group instead of OVA. The study protocol was approved by the Medical Ethics and Human Clinical Trial Committee at the Qingdao University and all the experiments described were performed in accordance with the regulations of the Centre of Animal Experiments of Qingdao University (Qingdao. China).

Tissue samples. Twenty-four hours subsequent to the last antigen challenge, the mice were sacrificed and the lungs were removed. The tissues from the left lung were directly obtained from the surgical suite and immediately fixed in $10 \%$ buffered formalin, prior to being embedded in paraffin. Following this, $5-\mu \mathrm{m}$ sections were prepared and stained with hematoxylin and eosin (H\&E). The thickness of the extracellular matrix (ECM) was determined subsequent to the staining of the tissue sections with $\mathrm{H} \& \mathrm{E}$. The average of 10 independent measurements was calculated for each section and the data were summarized.

Immunohistochemistry. The expression of TGF- $\beta 1$ was assessed using semi-quantitative immunohistochemistry. Following deparaffinization, the sections were incubated in $0.01 \mathrm{~mol} / 1$ citric acid buffer $(\mathrm{pH} 6.0$ ) for $15 \mathrm{~min}$ in a microwave for antigen retrieval. The sections were then cooled and incubated in $3 \mathrm{~g} / \mathrm{H}_{2} \mathrm{O}_{2}$ for $30 \mathrm{~min}$, in order to inactivate endogenous peroxidase, prior to being blocked with 1:10 normal horse serum for $30 \mathrm{~min}$. Following this, the supernatant was discarded. Primary anti-mouse TGF- $\beta 1$ (1:300 dilution) was added overnight at $4^{\circ} \mathrm{C}$ and then biotinylated goat anti-rat secondary antibody and streptavidin horseradish peroxidase were added to the slides. The slides were subsequently incubated for $30 \mathrm{~min}$ at room temperature. The staining was completed by incubation with diaminobenzidine chromogen solution at room temperature. The stained cells were then mounted and viewed using light microscopy.

Phase contrast microscopy. The phenotypic changes of the bronchial epithelial cells were assessed using phase contrast microscopy. The cultured bronchial epithelial cells were either treated with recombinant TGF- $\beta 1$ or left untreated (control) and the morphological changes were then visualized using phase contrast microscopy. The images were collected using a Nikon inverted microscope (Nikon Corp., Tokyo, Japan).

Small interfering RNA (siRNA) treatment. Bronchial epithelial cells were grown to $70 \%$ confluence on the culture dishes and the transient transfection was performed with specific stealth siRNA against Snail or control siRNA using Lipofectamine 2000, in accordance with the manufacturer's instructions. The target sequences for Snail were 5'-GCGAGCTGCAGGACTCTAA-3' (no. 1), and 5'-GCGAGT GGTTCTTCTGCGCTA-3' (no. 2); the scrambled control sequence was 5'-CACATGTTCCGATCTCGGC-3' (all synthesized by Qiagen N.V., Venlo, The Netherlands). Following $6 \mathrm{~h}$ incubation with the RNA-complex, the medium was replaced and $2 \mathrm{ml}$ fresh medium containing 10\% FBS was added. The cells were treated and harvested at the indicated times subsequent to the transfection.

Semi-quantitative reverse transcription-polymerase chain reaction $(q P C R)$. Total RNA was isolated from the cells using Trizol $^{\circledR}$ Reagent (Invitrogen Life Technologies, Carlsbad, CA, USA), according to the manufacturer's instructions. Total cellular RNA $(1 \mu \mathrm{g})$ was then reverse-transcribed into cDNA for the PCR amplification using a kit from 
Sigma. The sequences for the PCR primers used were: Snail sense, 5'-CAAGGAATACCTCAGCCTGG-3' and antisense, 5'-ATTCACATCCAGCACATCCA-3'; E-cadherin sense, 5'-TGGGTTATTCCTCCCATCAG-3' and antisense, 5'-TTTGTCAGGGAGCTCAGGAT-3'; vimentin sense, 5'-CGCTTCGCCAACTACATC-3' and antisense, 5'-GGTC AGGCTTGGAAACATC-3'; fibronectin sense, 5'-GCA CCAACTGACCTGAAG-3' and antisense, 5'-GCCACCATAA GTCCTGATAC-3'; and $\beta$-actin sense, 5'-CACCAACTGGGA CGACAT-3' and antisense, 5'-ACAG CCTGGATAGCAACG-3'. Amplification consisted of an initial 5 min incubation at $95^{\circ} \mathrm{C}$ and then 30 cycles of amplification with $30 \mathrm{sec}$ of denaturation at $95^{\circ} \mathrm{C}$, amplification for $30 \mathrm{sec}$ at $56^{\circ} \mathrm{C}$ and extension for $60 \mathrm{sec}$ at $72^{\circ} \mathrm{C}$. The final extension was set for $10 \mathrm{~min}$ at $72^{\circ} \mathrm{C}$. Data were expressed as the relative differences between the control and treated cells following normalization to $\beta$-actin expression.

Western blot analysis. Total cellular protein was extracted using a lysis buffer and quantified using protein quantification reagents from Bio-Rad (Hercules, CA, USA). Following this, $100 \mu \mathrm{g}$ of the protein was suspended in $5 \mathrm{X}$ reducing sample buffer, boiled for $5 \mathrm{~min}$, electrophoresed on $10 \%$ sodium dodecyl sulfate-polyacrylamide gel electrophoresis (SDS-PAGE) gels and then transferred to polyvinylidene difluoride membranes using electroblotting. The membranes were subsequently blocked in $1 \%$ bovine serum albumin (BSA)/0.05\% Tween-20/phosphate-buffered saline (PBS) solution overnight at $4^{\circ} \mathrm{C}$ and were then incubated with the primary antibody for $24 \mathrm{~h}$. A horseradish peroxidase-labeled immunoglobulin $\mathrm{G}$ ( $\mathrm{IgG}$ ) was used as the secondary antibody. Following this, the blots were developed by incubation in a chemiluminescent substrate and exposure to X-ray film. The blots were reprobed for GAPDH to ensure equal protein loading in each lane.

Statistical analysis. Data are expressed as the mean \pm standard deviation. Statistical comparisons of the data from the various groups were performed using the Student's t-test. Differences between groups were considered statistically significant at $\mathrm{P}<0.05$.

\section{Results}

Pathological alteration and TGF- $\beta 1$ expression in lung tissue. The airway wall in the mouse lung tissue was stained, prior to undergoing a histological examination. There was a little collagen deposition in the airway wall of the normal mice, while in the asthma model mice the deposition increased significantly, with an extensive distribution in the airway wall. TGF- $\beta 1$ protein was observed to be expressed in numerous types of lung cells in the model mice, including airway epithelial cells, fibroblasts, smooth muscle cells, vascular endothelial cells and infiltrative inflammatory cells, while there was a low expression of TGF- $\beta 1$ protein in the normal mice. TGF- $\beta 1$ protein levels were also assayed in the bronchoalveolar lavage fluid, which demonstrated that the TGF- $\beta 1$ levels were significantly higher in the asthmatic mice than those in the control group (Fig. 1).

TGF- $\beta 1$ induces mesenchymal phenotypic transformation in bronchial epithelial cells. Bronchial epithelial cells were

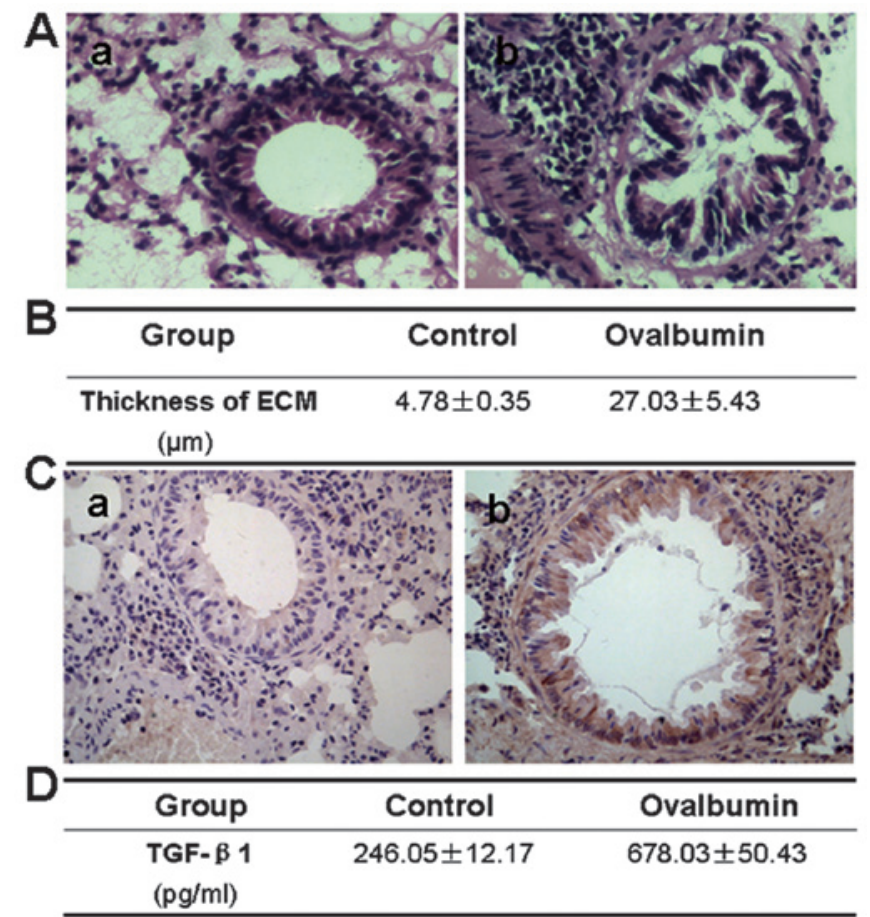

Figure 1. Airway remodeling and transforming growth factor- $\beta 1$ (TGF- $\beta 1$ ) expression in asthmatic mice. Lung tissue sections were obtained from mice $24 \mathrm{~h}$ subsequent to the final ovalbumin (OVA) challenge. (A) Hematoxylin and eosin (H\&E) staining of airway tissues in asthmatic mice. a) Control group; b) asthmatic group. (B) Morphometric parameters of the airway tissues in the control and asthmatic groups. (C) Expression of TGF- $\beta 1$ in the lung tissue was assessed using immunohistochemical staining. a) Control group; b) asthmatic group. (D) Enzyme-linked immunosorbent assay (ELISA) analysis of TGF- $\beta 1$ protein levels in bronchoalveolar lavage fluid. Magnification, x100. ECM, extracellular matrix.

activated with recombinant TGF- $\beta 1$ and the morphological changes were observed. The epithelial cells cultured in serum-free medium (control) showed a typical polygonal and cobblestone-monolayer morphology. In the bronchial epithelial cells activated with TGF- $\beta 1$, phenotypic changes were observed $48 \mathrm{~h}$ subsequent to TGF- $\beta 1$ activation. When compared with the control cells, the TGF- $\beta 1$-activated epithelial cells exhibited an elongated, spindle-shaped morphology, characteristic of fibroblasts. These morphological changes were associated with the loss of epithelial characteristics, such as E-cadherin, and with the acquisition of certain mesenchymal characteristics, including an increase in $\alpha$-SMA and vimentin expression. $\alpha$-SMA is considered a marker of myofibroblasts, which are the cell types present in EMT. The acquisition of a fibroblastic morphology and mesenchymal markers suggested that the epithelial cells had undergone EMT following treatment with TGF- $\beta 1$ (Fig. 2).

TGF- $\beta 1$ stimulation induces the upregulation of Snail. The zinc-finger transcription factor Snail is critical in the regulation of EMT during tumor progression and organ fibrosis. As a result of the correlation between Snail and EMT, we investigated whether the expression of Snail was upregulated by TGF- $\beta 1$ in bronchial epithelial cells. Compared with the untreated cells, TGF- $\beta 1$ increased Snail mRNA expression $12 \mathrm{~h}$ subsequent to treatment, with the highest level of expression being reached $48 \mathrm{~h}$ subsequent to treatment. The inducing 

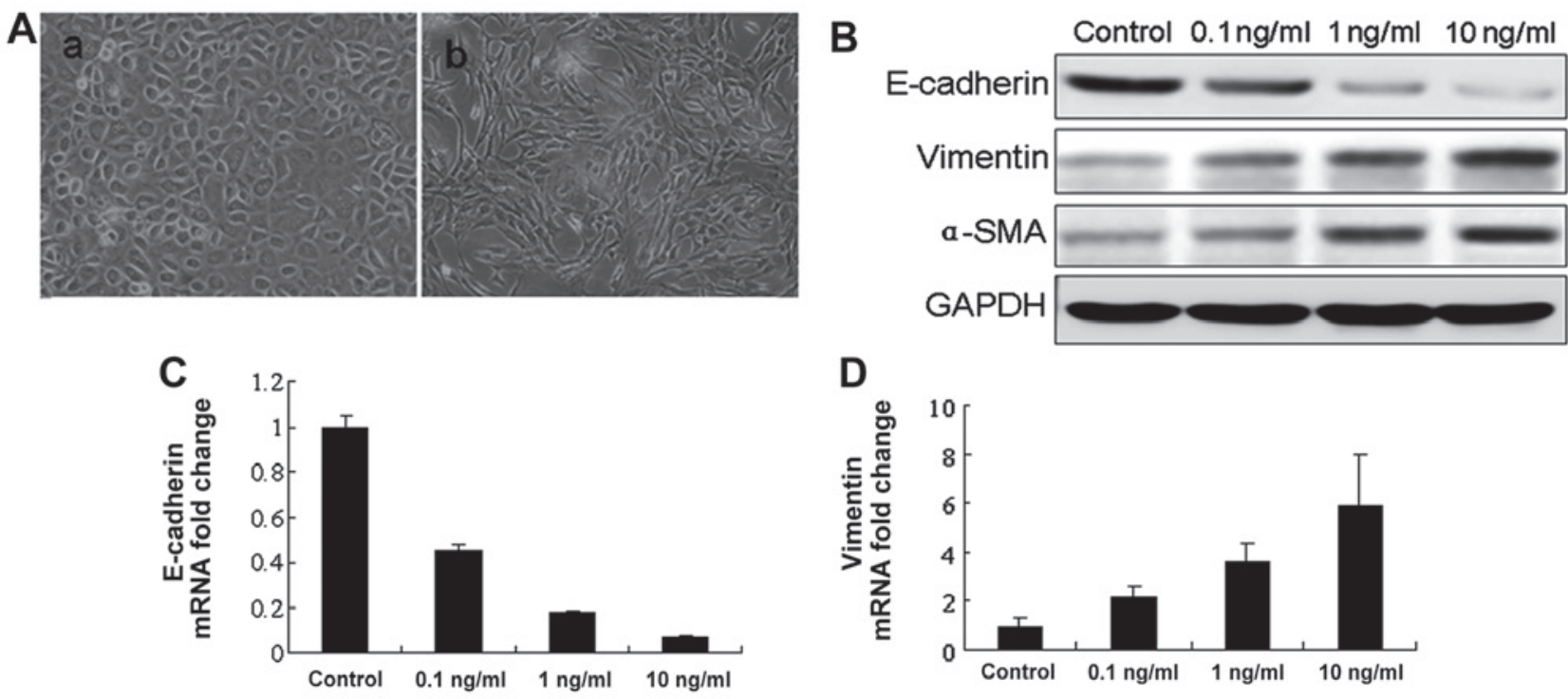

Figure 2. Bronchial epithelial cell transformation into myofibroblasts in response to transforming growth factor- $\beta 1$ (TGF- $\beta 1$ ) in vitro. Bronchial epithelial cells were incubated in the presence of TGF- $\beta 1(10 \mathrm{ng} / \mathrm{ml})$ for $48 \mathrm{~h}$. (A) The phenotypic changes were evaluated using phase contrast microscopy. a) Control epithelial cells; b) bronchial epithelial cells treated with TGF- $\beta 1$ (magnification, x40). (B) The expression of E-cadherin, vimentin and $\alpha$-smooth muscle actin $(\alpha$-SMA) was measured using western blot analysis. (C and D) The expression of E-cadherin and vimentin mRNA was measured using semi-quantitative reverse transcription-polymerase chain reaction analysis. GAPDH, glyceraldehyde 3-phosphate dehydrogenase.

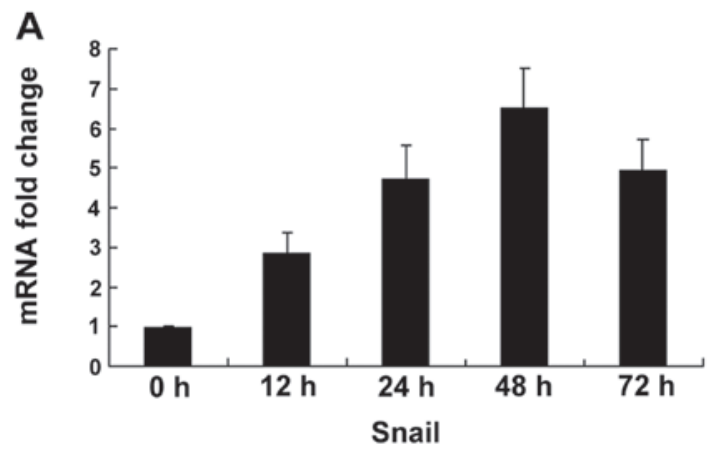

B

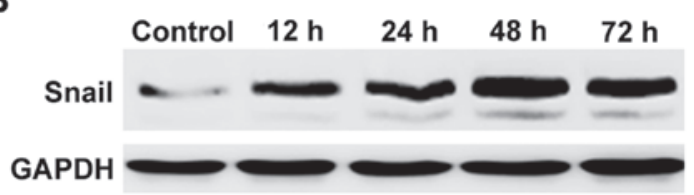

Figure 3. Transforming growth factor- $\beta 1$ (TGF- $\beta 1)$ increases Snail expression in cultured bronchial epithelial cells. Serum-starved bronchial epithelial cells were incubated with TGF- $\beta 1$ for up to $72 \mathrm{~h}$. (A) Total RNA was extracted and expression of Snail was measured using semi-quantitative reverse transcription-polymerase chain reaction analysis. (B) The expression of Snai protein was measured using western blot analysis. GAPDH, glyceraldehyde 3-phosphate dehydrogenase.

effects of TGF- $\beta 1$ on Snail protein levels were further demonstrated using western blot analysis (Fig. 3).

Silencing Snail blocks TGF- $\beta 1$-induced mesenchymal transformation in bronchial epithelial cells. To further elucidate whether Snail was involved in TGF- $\beta 1$-mediated EMT in bronchial epithelial cells, siRNAs were used to knock down the Snail gene in bronchial epithelial cells. As shown in Fig. 4A, siRNAi-Smad2\#1 caused a highly significant
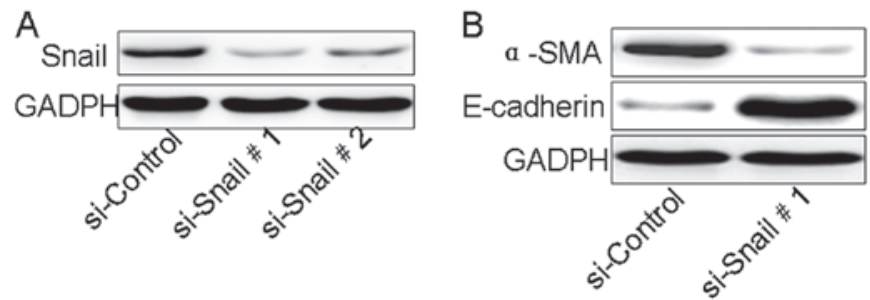

Figure 4. Downregulation of Snail reverses the epithelial-mesenchymal transition (EMT) of bronchial epithelial cells. Control small interfering RNA (siRNA), si-Snail\#1 and si-Snail\#2, two different siRNAs against Snail, were used. (A) Bronchial epithelial cells were transfected with siRNA-Snail, activated with transforming growth factor- $\beta 1$ (TGF- $\beta 1$ ) for $4 \mathrm{~h}$ and the transfection efficiency was confirmed. (B) Bronchial epithelial cells transfected with control siRNA or Snail-siRNA were assessed for the expression of $\alpha$-smooth muscle actin ( $\alpha$-SMA) and E-cadherin using western blot analysis. GAPDH, glyceraldehyde 3-phosphate dehydrogenase.

knockdown of Snail when compared with the control siRNA (Fig. 4A). Following the silencing of Snail in bronchial epithelial cells using siRNA-Snail, the protein expression levels of E-cadherin and $\alpha$-SMA in the total cell lysates were analyzed using western blot analysis. It was observed that the expression of epithelial markers was significantly increased, whereas the levels of mesenchymal markers were decreased (Fig. 4B).

TGF- $\beta 1$ increases fibronectin expression in bronchial epithelial cells. To evaluate our hypothesis and investigate whether the phenotypic changes observed following the treatment of bronchial epithelial cells with TGF- $\beta 1$ were due to ECM protein synthesis, we examined the expression of fibronectin in epithelial cells activated with TGF- $\beta 1$ at the transcriptional and translational levels. As expected, TGF- $\beta 1$ increased fibronectin mRNA production by bronchial epithelial cells in a dose- and time-dependent manner. The treatment of the 


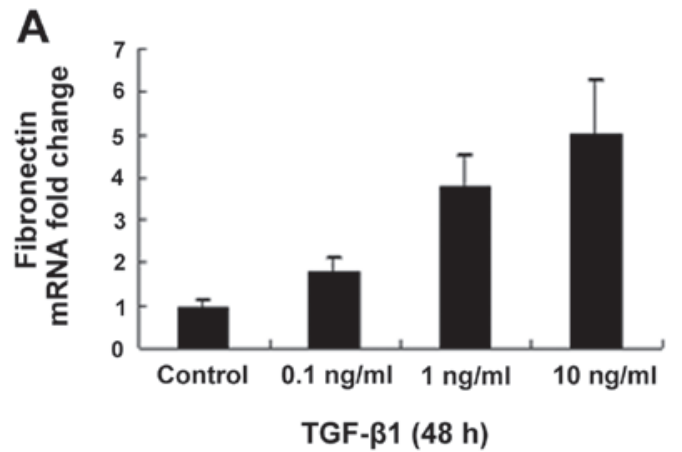

B

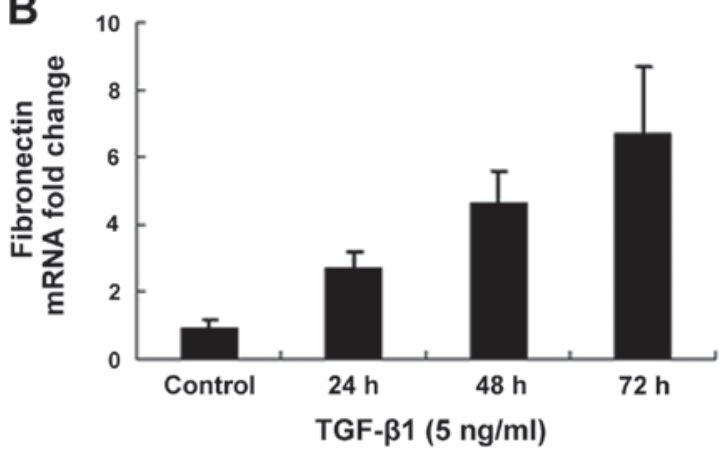

C

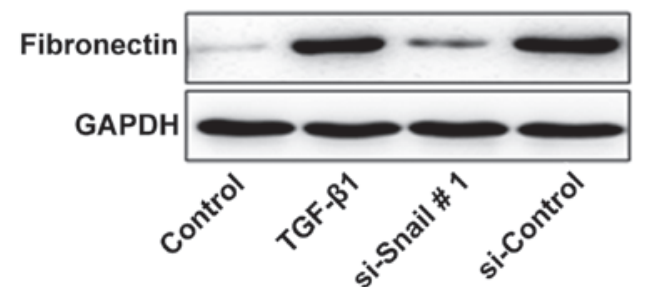

Figure 5. Transforming growth factor- $\beta 1$ (TGF- $\beta 1$ ) increases fibronectin expression in bronchial epithelial cells. Serum-starved bronchial epithelial cells were incubated with TGF- $\beta 1$ and (or) small interfering RNA (siRNA)-Snail. Total cellular mRNA and protein were extracted and subjected to reverse transcription-polymerase chain reaction and western blot analysis. (A) Dose-response of fibronectin expression. (B) Time-course of fibronectin expression. (C) Expression of $\alpha$-smooth muscle actin ( $\alpha$-SMA) in bronchial epithelial, cells measured using western blot analysis. GAPDH, glyceraldehyde 3-phosphate dehydrogenase.

bronchial epithelial cells with siRNA-Snail prior to TGF- $\beta 1$ stimulation significantly inhibited the expression of fibronectin compared with the control cells (Fig. 5).

\section{Discussion}

In this study, substantially thickened lung tissue and extensive fibrosis were observed in OVA-sensitized mice, which was interrelated with TGF- $\beta 1$ expression in bronchoalveolar lavage fluid. In vitro experiments demonstrated that bronchial epithelial cells responded to TGF- $\beta 1$ by transforming into myofibroblasts, indicating that these cells are capable of initiating and modulating the expression of epithelial markers to mesenchymal markers, in order to undergo EMT. Due to the fact that bronchial epithelial cells are able to transform into a fibroblast-like phenotype and secrete ECM compounds in response to TGF- $\beta 1$, it is possible that epithelial cells have a function in peribronchial fibrosis and promote airway remodeling in asthma.
The growth factor TGF- $\beta 1$ contributes to the development of fibroblastic foci in the bronchial region, and a number of studies have focused on the role of TGF- $\beta 1$ as the key promoter of peribronchial fibrosis $(12,18)$. However, the precise mechanisms responsible for the formation of fibroblastic foci are unknown and the origin of myofibroblasts, critical elements in the process of fibrosis, is not clearly understood. It has been suggested that epithelial cells, through the process of EMT, may be a possible source of myofibroblasts (5). Our results have shown that bronchial epithelial cells underwent a transition from a typical epithelial morphology to fibroblast-like cells following TGF- $\beta 1$ treatment, which was accompanied by the increased expression of $\alpha$-SMA and another mesenchymal marker, vimentin. Of note, TGF- $\beta 1$ was unable to induce EMT without disrupting the integrity of cell-cell contact, indicating the involvement of E-cadherin in TGF- $\beta 1$-mediated EMT (19). E-cadherin is the main component of adhesive junctions and is important in the assembly of junctional complexes and the maintenance of epithelial cell morphology. The downregulation of E-cadherin has been accepted as a characteristic of EMT (20). In the present study, the data demonstrated that bronchial epithelial cells underwent a transition from an epithelial to a mesenchymal phenotype following activation with TGF- $\beta 1$, which was characterized by a decrease in E-cadherin expression and an increase in vimentin and $\alpha$-SMA expression. Furthermore, TGF- $\beta 1$ treatment caused a significant change in bronchial epithelial cell morphology, with a transition from a typical epithelial morphology to a mesenchymal spindle-shaped morphology.

The E-cadherin suppressor, Snail, has been shown to be a key regulator of EMT in normal development and tumor progression (21). To the best of our knowledge, we have demonstrated for the first time that the Snail transcription factor was upregulated and that, subsequently, E-cadherin was downregulated during the EMT of bronchial epithelial cells. The increasing expression levels of the mesenchymal markers vimentin and $\alpha$-SMA were consistent with the TGF- $\beta 1$-induced upregulation of Snail. Our interest in Snail was further enhanced by the results from the knockdown experiments, due to the fact that the downregulation of Snail by a siRNA-expressing vector reversed the EMT of bronchial epithelial cells in response to TGF- $\beta 1$ treatment. These results demonstrated that the role of TGF- $\beta 1$ as an inducer of EMT was, at least in part, dependent on the functions of Snail.

Airway remodeling is one of the pathophysiological characteristics of asthma, and its main pathological changes include subepithelial fibrosis formation and increased collagen deposition on the airway wall (22). In order to confirm the effect of EMT on peribronchial fibrosis, we showed that TGF- $\beta 1$ increased fibronectin mRNA production by bronchial epithelial cells in a dose- and time-dependent manner; however, Snail siRNA transfection suppressed fibronectin protein expression in TGF- $\beta 1$-treated bronchial epithelial cells. These data further indicated that EMT was a key factor in peribronchial fibrosis and promoted airway remodeling in asthma.

Results of the present study showed that the concentration of TGF- $\beta 1$ in the bronchoalveolar lavage fluid was lower than that used in vitro to treat bronchial epithelial cells. This may have been due to the natural differences between in vivo and in vitro experiments, with the latter being acute and artificial. 
In addition, a number of other factors may contribute to the effect.

In conclusion, the present study showed that bronchial epithelial cells stimulated by TGF- $\beta 1$ exhibited phenotypic and molecular alterations, characteristic of EMT, through the upregulated expression of Snail, a transcription factor involved in EMT. We also showed that myofibroblasts originating from bronchial epithelial cells may contribute to peribronchial fibrosis in asthma. These results may enhance our understanding of the molecular mechanisms underlying the pathogenesis of peribronchial fibrosis, which may provide opportunities to prevent and treat asthma effectively.

\section{Acknowledgements}

This study was supported by the Natural Science Foundation of Shandong Province (no. Y2007C113) and the Science and Technique Foundation of Shandong Province (no. 2010GWZ20216).

\section{References}

1. Schlender A, Alperin PE, Grossman HL and Sutherland ER: Modeling the impact of increased adherence to asthma therapy. PLoS One 7: e51139, 2012.

2. Di Giampaolo L, Quecchia C, Schiavone C, Cavallucci E, Renzetti A, Braga M and Di Gioacchino M: Environmental pollution and asthma. Int J Immunopathol Pharmacol 24 (Suppl 1): S31-S38, 2011.

3. Yamauchi K and Inoue H: Airway remodeling in asthma and irreversible airflow limitation - ECM deposition in airway and possible therapy for remodeling. Allergol Int 56: 321-329, 2007.

4. Epperly MW, Guo H, Gretton JE and Greenberger JS: Bone marrow origin of myofibroblasts in irradiation pulmonary fibrosis. Am J Respir Cell Mol Biol 29: 213-224, 2003.

5. Yanez-Mo M, Lara-Pezzi E, Selgas R, et al: Peritoneal dialysis and epithelial-to-mesenchymal transition of mesothelial cells. $\mathrm{N}$ Engl J Med 348: 403-413, 2003.

6. Lv ZD, Na D, Ma XY, Zhao C, Zhao WJ and Xu HM: Human peritoneal mesothelial cell transformation into myofibroblasts in response to TGF- $\beta 1$ in vitro. Int J Mol Med 27: 187-193, 2011.

7. Lv ZD, Kong B, Li JG, Qu HL, Wang XG, Cao WH, Liu XY, Wang Y, Yang ZC, Xu HM and Wang HB: Transforming growth factor- $\beta 1$ enhances the invasiveness of breast cancer cells by inducing a Smad2-dependent epithelial-to-mesenchymal transition. Oncol Rep 29: 219-225, 2013.
8. Weber CE, Li NY, Wai PY and Kuo PC: Epithelial-mesenchymal transition, TGF- $\beta$, and osteopontin in wound healing and tissue remodeling after injury. J Burn Care Res 33: 311-318, 2012.

9. Carew RM, Wang B and Kantharidis P: The role of EMT in renal fibrosis. Cell Tissue Res 347: 103-116, 2012.

10. Grgic I, Duffield JS and Humphreys BD: The origin of interstitial myofibroblasts in chronic kidney disease. Pediatr Nephrol 27: 183-193, 2012.

11. Chapman HA: Epithelial-mesenchymal interactions in pulmonary fibrosis. Annu Rev Physiol 73: 413-435, 2011.

12. Qu ZH, Yang ZC, Chen L, Lv ZD, Yi MJ and Ran N: Inhibition airway remodeling and transforming growth factor- $\beta 1 / \mathrm{Smad}$ signaling pathway by astragalus extract in asthmatic mice. Int J Mol Med 29: 564-568, 2012.

13. Reinke LM, Xu Y and Cheng C: Snail represses the splicing regulator epithelial splicing regulatory protein 1 to promote epithelial-mesenchymal transition. J Biol Chem 287: 36435-36442, 2012.

14. Lander R, Nordin K and LaBonne C: The F-box protein Ppa is a common regulator of core EMT factors Twist, Snail, Slug, and Sip1. J Cell Biol 194: 17-25, 2011.

15. Yu LX, Zhou L, Li M, Li ZW, Wang DS and Zhang SG: The Notch1/cyclooxygenase-2/Snail/E-cadherin pathway is associated with hypoxia-induced hepatocellular carcinoma cell invasion and migration. Oncol Rep 29: 362-370, 2013.

16. Myong NH: Loss of E-cadherin and acquisition of vimentin in epithelial-mesenchymal transition are noble indicators of uterine cervix cancer progression. Korean J Pathol 46: 341-348, 2012.

17. Li H, Wang H, Wang F, Gu Q and Xu X: Snail involves in the transforming growth factor $\beta 1$-mediated epithelial-mesenchymal transition of retinal pigment epithelial cells. PLoS One 6: e23322, 2011.

18. Qin XJ, Zhang GS, Zhang X, Qiu ZW, Wang PL, Li YW, Li W, Xie QM, Ke YH, Lee JJ and Shen HH: Protein tyrosine phosphatase SHP2 regulates TGF- $\beta 1$ production in airway epithelia and asthmatic airway remodeling in mice. Allergy 67: 1547-1556, 2012.

19. Yook JI, Li XY, Ota I, Fearon ER and Weiss SJ: Wnt-dependent regulation of the E-cadherin repressor snail. J Biol Chem 280: 11740-11748, 2005.

20. Franz M, Spiegel K, Umbreit C, et al: Expression of Snail is associated with myofibroblast phenotype development in oral squamous cell carcinoma. Histochem Cell Biol 131: 651-660, 2009.

21. Ohkubo T and Ozawa M: The transcription factor Snail downregulates the tight junction components independently of E-cadherin downregulation. J Cell Sci 117: 1675-1685, 2004.

22. Zhang WX and Li CC: Airway remodeling: a potential therapeutic target in asthma. World J Pediatr 7: 124-128, 2011. 